\title{
Health disparities and infertility: impacts of state-level insurance mandates
}

\author{
Marianne Bitler, Ph.D., ${ }^{\mathrm{a}}$ and Lucie Schmidt, Ph.D. ${ }^{\mathrm{b}}$ \\ ${ }^{a}$ Public Policy Institute of California, San Francisco, California, and Labor and Population RAND Corporation, Santa Monica, \\ California; and ${ }^{\mathrm{b}}$ Economics Department, Williams College, Williamstown, Massachusetts
}

\begin{abstract}
Objective: To determine whether important racial, ethnic, or socioeconomic status (SES) health disparities exist in infertility, impaired fecundity, or infertility treatment.

Design: Four waves of the National Survey of Family Growth (NSFG) were pooled. Measures were compared across various race/ethnicity, education, and age groups.

Participant(s): Data for 31,047 women 15-44 years old from the NSFG were pooled.

Intervention(s): Outcomes were compared by whether the women's states of residence had a mandate in place (at least 1 year before the interview) to compel insurers to cover or offer to cover infertility treatment.

Main Outcome Measure(s): Infertility status, impaired fecundity, ever having sought infertility treatment.

Result(s): Infertility is more common for non-Hispanic black women, non-Hispanic other race women, and Hispanic women than for non-Hispanic white women, and both infertility and impaired fecundity are more common for high school dropouts and high school graduates with no college than for 4-year college graduates, and for older women compared with women 29 and younger. Older women, non-Hispanic white women, and women who are more educated (with at least some college) are more likely to have ever received treatment. No evidence has been found that the racial, ethnic, or education disparities are ameliorated by the health insurance mandates.

Conclusion(s): Racial, ethnic, and educational disparities exist in infertility status and treatment, and educational disparities in impaired fecundity. More study of the impact of infertility treatment mandates on these disparities is needed. (Fertil Steril ${ }^{\circledR} 2006 ; 85: 858-65$. (02006 by American Society for Reproductive Medicine.)
\end{abstract}

Key Words: Infertility treatment, infertility, impaired fecundity, health disparities, health insurance mandates

A large volume of research spanning the United States and many other countries has demonstrated the existence of racial, ethnic, and socioeconomic (SES) disparities in health status, access to health care, and utilization of health care services. One important area of reproductive health where disparities have not been examined is infertility. Infertility affects up to $10 \%$ of women of childbearing age (1), yet little research has examined differentials in either the prevalence of infertility or access to infertility treatment across different racial, ethnic, or SES groups.

In some other countries, the public health insurance system pays for some or all the costs of infertility treatment. In the United States, however, the bulk of expenditures are borne by the infertile couple, and many private insurance policies do not cover infertility treatment. These treatments can also be quite expensive, particularly when costs of higher risk multiple births are included (2). A 1992 study suggested that a successful birth after use of in vitro fertilization (IVF) would have cost between $\$ 44,000$ and $\$ 212,000$ (3). Because of the expense of receiving treatment, higher SES women receive the bulk of infertility treatments.

Received May 2, 2005; revised and accepted November 29, 2005.

Supported by National Institute of Child Health and Human Development (R03 HD046485 [Bitler] and R03 HD047544 [Schmidt]).

Reprint requests: Marianne P. Bitler, Ph.D., Public Policy Institute of California, 500 Washington Street, Suite 800, San Francisco, California 95111 (FAX: 1-415-291-4428; E-mail: bitler@ppic.org).
In response to concerns about the expense of infertility treatment, 15 states have passed mandates compelling health insurance companies to provide some form of infertility benefit. Additional states have ongoing legislative advocacy efforts in this area, and similar bills have also been introduced at the federal level. Given the continuing efforts to expand coverage, it is important to understand how the effect of these expansions may differ by race or SES.

This article uses pooled waves of the National Survey of Family Growth spanning 1982-2002 to examine the prevalence of infertility and impaired fecundity, the use of infertility treatment, and how infertility treatment is affected by state laws mandating infertility insurance benefits. We control for a series of individual and state level characteristics known to influence fertility behavior and infertility, and then interact our key variable-whether the woman's state of residence has an infertility mandate in place-with indicators for race/ethnicity, educational attainment (our SES measure), and age.

\section{Infertility and Impaired Fecundity in the United States}

Demographers using the National Survey of Family Growth have used two main definitions to classify whether women are having difficulties in childbearing. Infertility is only defined for women who are currently married or cohabiting, and is defined for those women as the condition of being 
unable to conceive after 12 or more consecutive months of unprotected intercourse. Impaired fecundity, however, applies to women of any marital or cohabiting status. Those who are nonsurgically sterile, who report having problems conceiving or carrying a pregnancy to term, as well those who are unable to conceive after 3 years of unprotected intercourse fall into this category (1). ${ }^{1}$ Potential problems can be found with each of these definitions (see (5) for a complete discussion). One major issue is that the trends in these two definitions appear to be diverging in recent years. Chandra and Stephen report that although the percentage of women with impaired fecundity has increased significantly between 1988 and 2002, the percentage of women with infertility has decreased significantly (6).

Although a great deal of research has documented disparities in health status by race, ethnicity, and SES, less is known about differentials in fertility and fecundity by these factors. However, some evidence has been found that the increase in impaired fecundity between 1982 and 1995 in the United States occurred across almost all subgroups of women, including along the dimensions of marital status, income, education, race, and ethnicity (1).

\section{Medical Assistance for Infertility/Impaired Fecundity}

Of the 6.2 million women with impaired fecundity in 1995, 2.7 million (44\%) had ever sought treatment (1). As a result of the high (and often uninsured) costs associated with treatment, however, medical assistance for infertility is sought primarily by women and couples that are white, college-educated, and affluent. Women with private health insurance coverage were 50\% more likely to have received services, as were women with income more than $300 \%$ of the poverty line (7). It is widely believed that an unmet need exists for infertility services, especially among those with lower incomes and lower levels of education (8).

\section{State Mandates to Insurers Regarding Infertility Treatment}

The first state-level infertility insurance mandate was enacted by West Virginia in 1977. Since that time, 14 other states have passed mandates, and additional states have ongoing legislative advocacy efforts in this area. A mandate "to cover" requires that health insurance companies provide coverage of infertility treatment as a benefit included in every policy bought by firms. A mandate "to offer" requires that health insurance companies make available to firms for purchase a policy which offers coverage of infertility treatment. In addition, some mandates cover all health plans, whereas others either exclude health maintenance organizations (HMOs) or only cover HMOs. Finally, some mandates

\footnotetext{
We have modified the standard demographic definition to include the non-surgically sterile as having impaired fecundity and the surgically sterile as not having impaired fecundity. Recent work advocates using the World Health Organization definition of trying to get pregnant for 24 months (4), however this measure is not available in our data.
}

\section{TABLE 1}

\section{State-mandated infertility insurance.}

\begin{tabular}{|lc|}
\hline State & Year law enacted \\
\hline Arkansas & $1987^{\mathrm{a}}$ \\
California & $1989^{\mathrm{a}}$ \\
Connecticut & 1989 \\
Hawaii & 1987 \\
Illinois & 1991 \\
Louisiana & 2001 \\
Maryland & 1985 \\
Massachusetts & 1987 \\
Montana & 1987 \\
New Jersey & 2001 \\
New York & $1990^{\mathrm{a}}$ \\
Ohio & 1991 \\
Rhode Island & 1989 \\
Texas & 1987 \\
West Virginia & $1977^{\mathrm{a}}$ \\
\hline
\end{tabular}

Note: Data come from Resolve (http://www.resolve.org) and state laws (see Appendix A of Schmidt (9)).

a Arkansas, California, New York, and West Virginia first passed mandates in the years shown. These mandates were subsequently revised, but remained in place.

Bitler. Infertility disparities and insurance mandates. Fertil Steril 2006.

exclude coverage of IVF, which is one of the most expensive treatments available for infertility. Table 1 provides a list of the states with mandates currently in place and the date the mandates were enacted. ${ }^{2}$

Using a differences-in-differences approach and Vital Statistics data for 1985-1999, Schmidt finds that the mandates increase first birth rates for all women 35 and older by $32 \%$, but finds no evidence of an effect of the mandates on the first birth rates of black women (10). Two other recent studies use clinic data from 1998 and find that states with required coverage for IVF have the highest rates of IVF utilization $(11,12)$. Hamilton and McManus develop a theoretical model of the market for IVF and use data from clinics at the Metropolitan Statistical Area level to test their model's predictions (unpublished observation). Using data from 19952000, they confirm the findings reported in (11) and (12) that a mandate increases IVF utilization rates. They also find that clinics are attracted to areas where women are more educated and wealthier, but find no evidence that clinics are attracted to places where mandates are in effect.

Another recent study looks at the impacts of infertility mandates on twin births and mixed-sex twin births (13), finding that for women over 35 , living in a mandate state is associated with a statistically significant increase in twin

\footnotetext{
2 Detailed information on these mandates, including any further restrictions placed on coverage, can be found in Schmidt (9)
} 


\section{TABLE 2}

\section{Summary statistics for infertility, impaired fecundity, and infertility treatment.}

\begin{tabular}{|lccc|}
\hline Sample & $\begin{array}{c}\text { 12-month } \\
\text { infertility }\end{array}$ & $\begin{array}{c}\text { Impaired } \\
\text { fecundity }\end{array}$ & $\begin{array}{c}\text { Ever had infertility } \\
\text { treatment }\end{array}$ \\
\hline Married now sample & 0.077 & 0.178 & - \\
Sex ever after menarche sample & $\begin{array}{c}0.047 \\
\text { Married now }\end{array}$ & $\begin{array}{c}0.151 \\
\text { Married now }\end{array}$ & $\begin{array}{c}\text { Sex ever } \\
\text { Sample is divided by }\end{array}$ \\
Race/ethnicity & 0.069 & 0.177 & 0.158 \\
White non-Hispanic & 0.120 & 0.198 & 0.107 \\
Black non-Hispanic & 0.100 & 0.182 & 0.128 \\
Other race non-Hispanic & 0.092 & 0.171 & 0.115 \\
Hispanic & & & 0.099 \\
Education level & 0.099 & 0.210 & 0.144 \\
High school dropout & 0.082 & 0.183 & 0.155 \\
High school graduate & 0.066 & 0.176 & 0.182 \\
Some college & 0.067 & 0.152 & 0.075 \\
College graduate & & 0.106 & 0.185 \\
Maternal age & 0.064 & 0.166 & 0.201 \\
Age 29 or less & 0.080 & 0.230 & \\
Age 30-34 & 0.084 & & \\
Age 35-44 & & & \\
\hline
\end{tabular}

Note: Values are weighted means. Column 1 means are for 12-month infertility, column 2 for impaired fecundity, and column 3 for ever having sought infertility treatment. Means in the top panel, first row are for women age 15-44 who are currently married; those in the top panel, second row are for women age 15-44 who ever had sex after menarche. Means in the bottom panels (by race/ethnicity, education level, and age) are for the married now sample for column 1 (12-month infertility measure) and column 2 (impaired fecundity measure) but are for the sex ever after menarche sample from column 3 (ever had infertility treatment).

Bitler. Infertility disparities and insurance mandates. Fertil Steril 2006.

births (relative to singletons), and in mixed-sex twin births (relative to same sex twins or singletons). Among twins, being born to an older mother in a mandate state is associated with being a lower birth weight, shorter gestation infant with a lower Apgar score.

\section{MATERIALS AND METHODS}

We pooled data from the 1982, 1988, 1995, and 2002 cycles of the National Survey of Family Growth (NSFG). The NSFG is a periodic survey conducted by the National Center for Health Statistics (NCHS), and surveys the civilian, noninstitutionalized population of women 15-44 years old. The main purpose of the survey is to provide reliable national data on marriage, divorce, contraception, and the health of women and infants in the United States. Most important for our analysis, the NSFG is the only source of information over time about infertility, impaired fecundity, and use of infertility treatment in the United States. We use public-use data merged with state of residence identifiers at NCHS's Research Data Center. This allows us to link our measure of access to infertility treatment-whether an individual lived in a state that mandated coverage of infertility treatment- to the individual-level data.

We also link other contextual measures such as state-level demographics, labor market conditions, and public assistance gen- erosity. For each woman, we link the nonmandate contextual variables by the year in which her interview was completed. The mandate variables are set to 1 if the mandate had been implemented in the year before the woman was interviewed.

We first present summary statistics for our key outcomes of interest. Table 2 presents prevalence-type measures for three variables: infertility (defined as being unable to conceive despite having had sex with the same partner for 12 months without contraception or pregnancy), impaired fecundity (defined as having trouble conceiving or carrying a child to term, having been told by a doctor not to become pregnant, having had sex for 36 months with the same partner with no contraception or successful pregnancy, or being noncontraceptively sterile), and having ever sought a doctor's help to become pregnant or avoid miscarriage. ${ }^{3}$

\footnotetext{
${ }^{3}$ These measures are complicated by the fact that for those women classified as having fertility problems based on a fixed time period of unprotected sex, they can exit the state of infertility either by stopping intercourse or by becoming pregnant. The treatment measure is the share of women who report ever having sought treatment for infertility, a prevalence measure.

We also calculate the measures of infertility and impaired fecundity on the sample of married women, to be consistent with earlier literature using the NSFG. These latter two statistics are less straightforward prevalence measures, since the denominator is changing over time.
} 
The first two measures are given for two different samples, the sample of all women 15-44 years old who have ever had sex after menarche and the sample of married women. The infertility treatment measure is given only for women who have ever had sex after menarche. ${ }^{4}$ The lower panels of the table present means for all three measures by race/ethnicity (upper middle panel), educational attainment (lower middle panel), and age (lowest panel). In the lower panels of Table 2, the sample for infertility and impaired fecundity is women currently married, whereas the sample for the infertility treatment measure is women having had sex after menarche.

Several patterns are clear from Table 2. First, as would be expected given the broader definition, the prevalence of impaired fecundity is higher than the prevalence of infertility. Second, both infertility and impaired fecundity are more common among non-white women, among less educated women, and among older women (with the sole exception that Hispanic women are less likely than white non-Hispanic women to have impaired fecundity). However, use of treatment is less common among non-white women and women with lower levels of education, and much more common among older women.

We then turn to multivariate logistic regressions, estimated using Stata 9.1 (StataCorp LP, College Station, TX). We first examine racial, ethnic, and SES differences in infertility status, impaired fecundity, and ever having sought infertility treatment. We then consider whether living in a state with an infertility mandate has differential effects by race and ethnicity, SES (education), or age. We control for other characteristics (age, demographic and economic characteristics of the woman's state of residence, state and year fixed effects). We weight the regressions to be populationrepresentative and adjust the standard errors for arbitrary correlation within state-by-year cell and for heteroscedasticity. $^{5}$

The regressions for the prevalence measure of ever having had a visit for infertility treatment are run on the sample of

\footnotetext{
${ }^{4}$ Note that the lower panels of Table 2 and the regression results reported in Table 3, Column 2 estimate the determinants of impaired fecundity for only the sample of women married now to be comparable to the results for infertility status.

${ }^{5}$ The NSFG data are complex sample surveys. Our method of adjusting the standard errors is as conservative as standard survey methods for our estimates. Theoretically, this would be likely because the primary sampling units (PSUs) or clusters for the NSFG data are typically counties or MSAS, and are contained within states. Thus, allowing for arbitrary correlations within state-by-year cell should lead to larger standard errors than if we simply allowed such correlations within PSU, unless the error term covaries negatively within state. Empirically, we have also estimated our key regressions using one of Stata 9.1's survey method estimators, a version of logistic regression which adjusts the standard errors using Taylor series linearization methods for both stratification and clustering within PSU.

These estimates are close to those reported in the paper. Because our key mandate variable only varies at the state-by-year level, adjusting variance estimates using survey methods still may overstate significance. The alternate survey method results are available on request.
}

women ever having had sex after menarche. This prevalence measure is asked of all women, and $7.9 \%$ of women who are not currently married but have ever had sex after menarche report having had such a visit. Thus, we would miss women who have gotten treatment were we to restrict the treatment regressions to the sample of women who were currently married. The regressions for the measures of current infertility status or impaired fecundity are run on the sample of currently married women.

Approval to conduct this study was obtained from the RAND Corporation's Human Subjects Protection Committee, the Institutional Review Board.

\section{RESULTS}

Odds ratios from logistic regressions of the determinants of 12-month fertility can be found in column 1 of Table 3 (along with the marginal effect for the any infertility mandate variable). As would be expected, age has a strong effect on the probability of being infertile, with teenagers being the least likely to experience infertility and the probability increasing significantly by age. ${ }^{6}$ In addition, strong evidence exists about the disparities by both race and SES (as proxied for by educational attainment) in infertility. All non-white racial and ethnic groups (black, other race, and Hispanic) are significantly more likely to experience infertility than the omitted group of whites, and both high school dropouts and high school graduates are significantly more likely to experience infertility than four-year college graduates.

Column 2 of Table 3 presents results from a similar regression that looks at determinants of impaired fecundity. In general, the patterns are similar for the two variables. Both age and education have strong effects on the likelihood of infertility and impaired fecundity. However, although the odds ratios for blacks and other race women are both greater than 1 , these racial disparities in impaired fecundity are not statistically significant.

Several state-level variables also have a significant effect on fertility problems. The percent of the state population that is Hispanic has a significant negative effect on infertility status that persists even after controlling for individual race/ ethnicity. Some evidence has also been found that after controlling for race and SES, states that are better off financially are less likely to experience infertility: The percent of the population below the federal poverty line has a positive and significant effect on infertility, but has no significant effect on impaired fecundity. No evidence has indicated that the statelevel mandates have an effect on the likelihood that a woman is categorized with either infertility or impaired fecundity-the odds ratio is close to 1 and insignificant.

\footnotetext{
${ }^{6}$ Note that part of this age discrepancy is due to the fact that women who are likely to delay childbearing are less likely to be married young and thus less likely to appear in the sample while young but may appear while older.
} 
Characteristics associated with infertility, impaired fecundity, and infertility treatment.

\begin{tabular}{|c|c|c|c|}
\hline & 12-month infertility & Impaired fecundity & $\begin{array}{l}\text { Ever had infertility } \\
\text { treatment }\end{array}$ \\
\hline Any infertility mandate & $0.9904(0.1272)$ & $1.0905(0.1167)$ & $1.0011(0.0821)$ \\
\hline Age $15-19$ & $0.3308^{a}(0.1581)$ & $0.4363^{a}(0.1387)$ & $0.1949^{a}(0.0282)$ \\
\hline Age 20-24 & $1.0403(0.1602)$ & $0.6788^{a}(0.0801)$ & $0.5039^{\mathrm{a}}(0.0429)$ \\
\hline Age 30-34 & $1.3150^{\mathrm{b}}(0.1416)$ & $1.4854^{a}(0.1214)$ & $1.6210^{\mathrm{a}}(0.1021)$ \\
\hline Age 35-39 & $1.3176^{b}(0.1452)$ & $1.9894^{a}(0.1845)$ & $1.7658^{\mathrm{a}}(0.1206)$ \\
\hline Age $40-44$ & $1.4398^{\mathrm{a}}(0.1631)$ & $2.6025^{a}(0.2001)$ & $1.8359^{\mathrm{a}}(0.1220)$ \\
\hline Black & $1.7215^{\mathrm{a}}(0.1539)$ & $1.0939(0.0781)$ & $0.7069^{\mathrm{a}}(0.0359)$ \\
\hline Other race & $1.4479^{b}(0.2424)$ & $1.1110(0.1359)$ & $0.7683^{\mathrm{b}}(0.0837)$ \\
\hline Hispanic & $1.2707^{b}(0.1506)$ & $0.9101(0.0793)$ & $0.8095^{\mathrm{b}}(0.0773)$ \\
\hline High school dropout & $1.5763^{\mathrm{a}}(0.1798)$ & $1.6716^{a}(0.1385)$ & $0.7497^{\mathrm{b}}(0.0534)$ \\
\hline High school graduate & $1.2802^{\mathrm{b}}(0.1544)$ & $1.3196^{a}(0.0973)$ & $0.8786^{\mathrm{b}}(0.0550)$ \\
\hline $\begin{array}{l}\text { Some college, no 4-year } \\
\text { degree }\end{array}$ & $1.0125(0.1108)$ & $1.2659^{\mathrm{a}}(0.0966)$ & $0.9757(0.0510)$ \\
\hline Metropolitan statistical area & $1.1397(0.1002)$ & $1.0683(0.0685)$ & $1.0682(0.0633)$ \\
\hline \% Hispanic & $0.9445^{\mathrm{b}}(0.0273)$ & $0.9939(0.0196)$ & $0.9769(0.0187)$ \\
\hline$\%$ black & $0.9430(0.0512)$ & $0.9970(0.0505)$ & $0.9546(0.0405)$ \\
\hline Medicaid eligibility threshold & $1.0013(0.0011)$ & $1.0001(0.0008)$ & $1.0006(0.0008)$ \\
\hline AFDC/TANF benefits & $1.0190(0.0693)$ & $1.0305(0.0552)$ & $0.9738(0.0439)$ \\
\hline Real median income & $1.0338(0.0233)$ & $0.9968(0.0145)$ & $1.0284^{\mathrm{C}}(0.0155)$ \\
\hline Unemployment rate & $0.0140(0.0702)$ & $0.2067(0.5915)$ & $7.4814(21.414)$ \\
\hline Employment growth rate & $0.2228(1.1668)$ & $0.0467(0.1349)$ & $0.0755(0.2270)$ \\
\hline Share of population under FPL & $269.85^{\mathrm{C}}(798.84)$ & $0.0383(0.0768)$ & $0.2757(0.5083)$ \\
\hline $\begin{array}{l}\text { Share of births to unmarried } \\
\text { women }\end{array}$ & $0.3739(0.8694)$ & 1.0907 (1.6820) & $2.6688(3.4323)$ \\
\hline Marginal effect of any mandate & $-0.0001(0.0089)$ & $0.0124(0.0155)$ & $0.0001(0.0098)$ \\
\hline Number of observations & 15,952 & 15,952 & 31,047 \\
\hline Log-likelihood & -4213.22 & -7186.58 & $-12,140.24$ \\
\hline Pseudo R-squared & 0.03 & 0.04 & 0.06 \\
\hline
\end{tabular}

Note: Each column presents odds ratios from a separate logistic regression or, for the mandate variable, the marginal effect. Regressions include state and year that fixed effects and are weighted. Standard errors adjusted for arbitrary correlation within state-by-year cell and heteroscedasticity. Levels of statistical significance: ${ }^{a}$ denotes significance at the $1 \%$ level; ${ }^{\mathrm{b}}$ at the $5 \%$ level; and ${ }^{\mathrm{c}}$ at the $10 \%$ level. Significance levels of odds ratios are from tests of logistic regression coefficients being zero. Marginal effect for any infertility mandate calculated for each observation and averaged across the sample, with standard errors calculated by the delta method. AFDC = Aid to Families with Dependent Children; TANF $=$ Temporary Assistance to Needy Families; FPL = Federal Poverty Level.

Bitler. Infertility disparities and insurance mandates. Fertil Steril 2006.

We next look at the determinants of a woman reporting that she has ever received infertility treatment. These results are presented in column 3 and are for the sample of all women who have had sex after menarche. Despite the evidence of higher likelihoods of fertility problems by race, ethnicity, and SES presented previously, women who are not white and women who are of lower SES are significantly less likely to report ever having received infertility treatment. Black women are $29 \%$ less likely to report ever having had infertility treatment than white women, and similar racial gaps exist (although slightly smaller in magnitude) for other race and Hispanic women.
Similarly, there are large differentials in reports of treatment by educational level. High school dropouts are $25 \%$ less likely to report treatment than college graduates, and high school graduates are $12 \%$ less likely to report treatment. No evidence has indicated that state-level characteristics significantly affect access to infertility treatment once state fixed effects are controlled for with one exception: women in states with higher real median income are significantly more likely to report having seen a doctor for infertility treatment. The odds ratio for the mandate variable is not statistically significant, suggesting that the mandates do not significantly affect the probability that a woman has ever received treatment. 


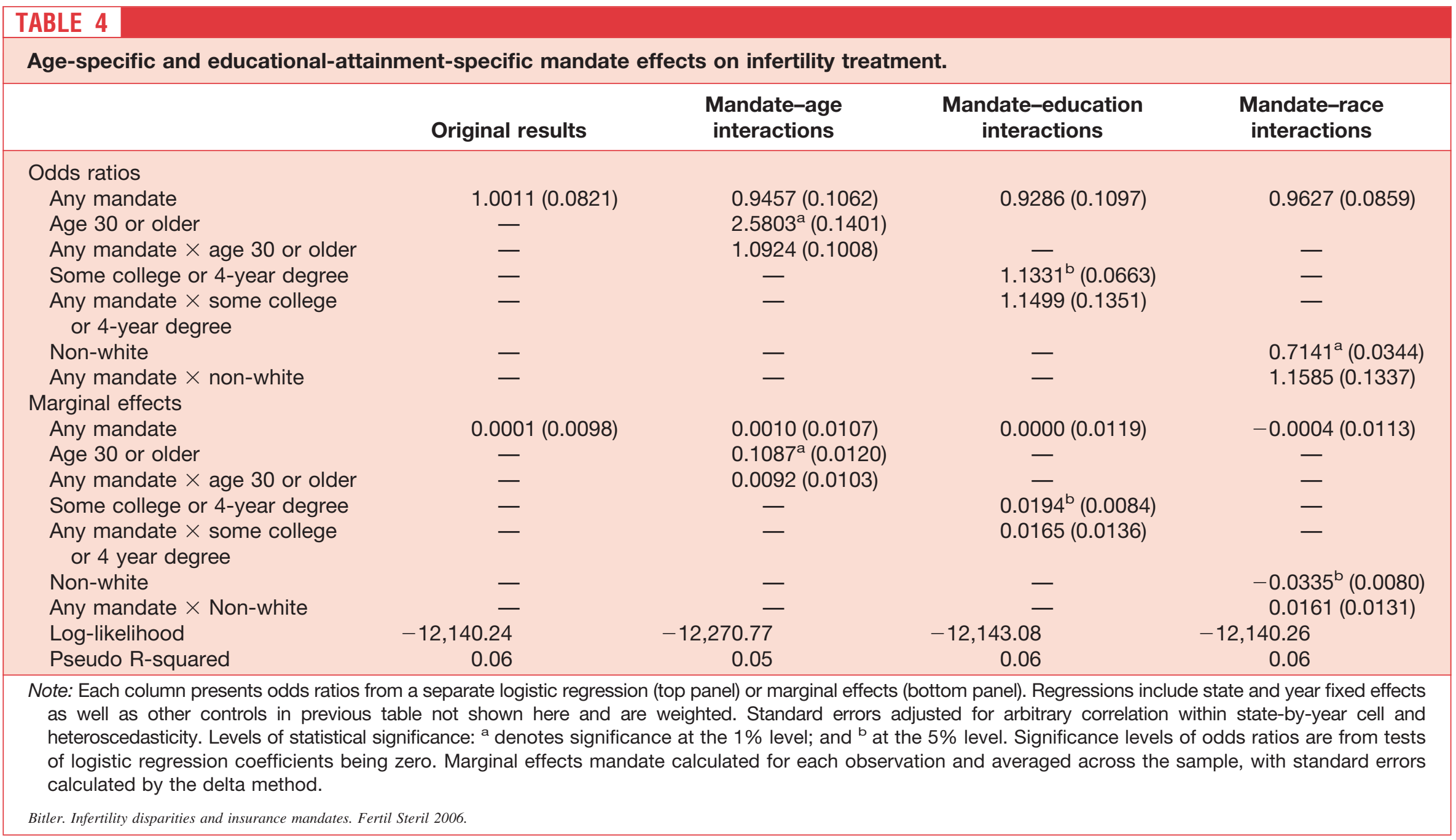


In Table 4, the regressions test whether the mandates have differential effects for different groups of women. In column 1, we use the odds ratio from column 3 of Table 3 for comparison. In column 2 , the mandates are interacted with an indicator for whether the woman is 30 or older, allowing the mandate to have differential effects on older vs. younger women. The top panel contains the odds ratios. Because the model is nonlinear and the marginal effects depend on the value of the various controls, however, it is necessary to calculate marginal effects to assess whether the interaction is significant. The marginal effect is calculated for each observation and averaged across the sample, with standard errors calculated by the delta method (14). These marginal effects are presented in the lower panel of the table. The results indicate no statistically significant evidence of any agevarying mandate effects.

In column 3, we allow for the mandates to have differential effects by SES (as proxied by educational level being some college or a 4-year degree). The rhetoric surrounding the push for mandated coverage of infertility treatment at either the state or federal level often involves expanding access to those groups who have been traditionally less likely to receive treatment. However, our results in column 3 indicate no evidence of expanded access. Again, the marginal effects are reported in the bottom panel of the tables. No evidence indicates that mandates increase the probability of treatment for women with varying levels of education, despite the strong statistical significance of the main education effect. Results in column 4 indicate similar patterns when we interact the mandates with an indicator for whether the woman is not white-again, no evidence indicates that the mandates have expanded access to these groups. Interestingly, a model with a three-way interaction between high education, any mandate, and age at least 30 (along with all the other two-way and one-way interactions) leads to a marginal effect on the three-way interaction of 0.0464 (0.0227), which is significant at the 5\% level. This finding also suggests that any impacts of the mandates in expanding access are confined to highly educated older women. ${ }^{7}$ This is consistent with the fact that private insurance coverage is more common for this group of women.

\section{DISCUSSION}

We find strong evidence of racial, ethnic, and SES disparities in both infertility status and access to treatment. Hispanic women and non-Hispanic black and other-race women, are significantly more likely to be infertile, but not significantly more likely to report impaired fecundity. One possible explanation for this discrepancy is that the impaired fecundity measure may be more subjective than the 12-month infertility measure. Although infertility is based on not becoming pregnant during a 12-month period of unprotected intercourse, one reason for being classified as impaired fecundity

\footnotetext{
${ }^{7}$ These results are available from the authors.
}

is thinking that one would have difficulty getting pregnant or carrying a pregnancy to term. This measure may be more likely influenced by external factors.

High school dropouts and high school graduates are also significantly more likely to report both infertility and impaired fecundity than their more highly educated counterparts. Hispanic women and non-Hispanic black and other-race women, as well as less-educated women, are all significantly less likely to have ever sought medical treatment to get pregnant or to prevent miscarriage.

However, we find no significant effects of the mandates on use of treatment for the overall sample. One possible explanation is due to the distinction between prevalence and incidence. Conceptually, what we would ideally like to analyze is the effect of a mandate on the probability that a woman has treatment in a given year (i.e., the flow of women into the pool of the treated). The variable we are analyzing is the stock of women who have ever received treatment. Even if the mandates increase the number of women who receive treatment in any given year, this increase may be small enough relative to the stock of women who ever obtained treatment that the estimated coefficient may not be statistically significant.

In addition, we find no evidence that these mandates have mitigated the disparities in treatment by race, ethnicity, or SES (as proxied by education). These findings are consistent with results from a survey done in Massachusetts, that suggest that even in a state with a comprehensive mandate, disparities in access to treatment exist along racial and SES dimensions (15). In fact, our three-way interaction results indicate a positive and statistically significant marginal effect of the mandates on highly educated women over 30 and older, which is additional evidence that despite the rhetoric of expanded access accompanying passage of the mandates, these laws may not be reducing existing disparities in treatment. The results from the three-way interactions may not be surprising given that highly educated women 30 and older are also the group most likely to have private insurance. Additional research is necessary to further explore why mandates do not reduce these disparities. In addition, future studies should investigate whether the mandates have affected the timing of infertility visits or the types of diagnoses and treatment obtained.

\section{REFERENCES}

1. Chandra A, Stephen EH. Impaired fecundity in the United States: 1982-1995. Fam Plann Perspect 1998;30:34-42.

2. Katz PP, Nachtigall R, Showstack J. The economic impact of the assisted reproductive technology. Nature Medicine 2002;8:S29-32.

3. Neumann PJ, Gharib, SD, Weinstein, MC. The cost of a successful delivery with in vitro fertilization. N Engl J Med 1994;331:239-43.

4. Larsen U. Research on infertility: which definition should we use? Fertil Steril 2005;83:846-52.

5. Chandra A, Stephen EH, Habbema D, te Velde ER. How long can you wait? Measurement challenges and health concerns related to infertility and delayed childbearing. In: Program of the annual meeting of Population Association of America, Minneapolis, MN, May 1-3, 2003. 
6. Chandra A, Stephen EH. Infertility and medical care for infertility: trends and differentials in national self-reported data. In: Program of the NICHD Conference on Health Disparities and Infertility. Bethesda, MD: NICHD, March 10-11 2005.

7. Stephen EH, Chandra A. Use of infertility services in the United States: 1995. Fam Plann Perspect 2000;32:132-7.

8. Chandra A, Mosher WD. The demography of infertility and the use of medical care for infertility. Infertility and Reproductive Medicine Clinics of North America 1994;5:283-96.

9. Schmidt L. Effects of infertility insurance mandates on fertility. In: Program of the Annual Meeting. Philadelphia, PA: Population Association of America, March 31-April 2, 2005.

10. Schmidt L. Infertility insurance mandates and fertility. Am Econ Rev Papers and Proceedings 2005;95:204-8.
11. Jain T, Harlow BL, Hornstein MD. Insurance coverage and outcomes of in vitro fertilization. N Engl J Med 2002;347:661-6.

12. Reynolds MA, Schieve LA, Jeng G, Peterson HB. Does insurance coverage decrease the risk for multiple births associated with reproductive technology? Fertil Steril 2003;80:16-23.

13. Bitler MP. The effects of increased access to infertility treatment on infant and child health: Evidence from health insurance mandates. In: Program of the Annual Meeting. Philadelphia, PA: Population Association of America, March 31-April 2, 2005.

14. Oehlert GW. A note on the delta method. American Statistician 1992; 46:27-9.

15. Jain T, Hornstein MD. Disparities in access to infertility services in a state with mandated insurance coverage. Fertil Steril $2005 ; 84: 221-3$ 